\title{
WATER MASERS TOWARD LOW-LUMINOSITY YOUNG STELLAR OBJECTS
}

\author{
MARK J CLAUSSEN \\ National Radio Astronomy Observatory, Socorro, NM, USA \\ KEVIN B. MARVEL \\ Owens Valley Radio Observatory, Big Pine, CA, USA \\ H. ALWYN WOOTTEN \\ National Radio Astronomy Observatory \\ Charlottesville, VA, USA \\ AND \\ BRUCE A. WILKING \\ University of Missouri, St. Louis, MO, USA
}

\begin{abstract}
A review of the importance of water maser observations toward young stellar objects (YSOs) is presented. Also, we present recent, differing types of observations of water masers near YSOs. Single antenna observations, taken regularly, characterize the variability of the masers and allow estimates of time and spatial scales. High resolution ( 1 mas) multi-epoch observations allow proper motions to be studied. Detailed analysis of such proper motions will allow the placement of the masers in the circumstellar (a disk) or near-stellar environment at the base of the outflow. Radio interferometric techniques are the best method of making estimates of the kinematics of the gas in these regions.
\end{abstract}

\section{Introduction}

Water maser emission at $22 \mathrm{GHz}$ is found to be a tracer of mass-loss activity in young stars of all masses (e.g., Rodríguez et al. 1980, Genzel \& Downes 1977). This mass loss occurs during an early, embedded phase of pre-mainsequence stars characterized by large quantities of circumstellar dust which completely absorbs visible light from the central object. The emergent energy from such YSOs is photospheric and disk radiation reprocessed by 
circumstellar dust and radiated at mid- to far-infrared wavelengths. But all too often the interest of astronomers is focussed on the spectacularly strong masers, with high-velocity features, that arise in flows near very massive YSOs, those which are forming O or B stars, e.g. the Orion water masers, or the masers near W49. The low-mass YSOs and their associated gas outflows have been well-studied, both observationally and theoretically (note the beautiful results at this meeting). So it is important to study the water masers around embedded sources of low luminosity. Although they may be generally weak, the sensitivity of large radio telescopes are such that telescope time is no longer prohibitive in the study of weak maser sources.

Water masers, whether near high-mass or low-mass stars, have long been known to be variable. Recent surveys of low-mass YSOs for $\mathrm{H}_{2} \mathrm{O}$ maser emission have demonstrated that while such emission is common, it is also highly variable; e.g., Comoretto et al. (1990); Felli, Palagi, \& Tofani (1992); Wilking et al. (1994a); Xiang \& Turner (1992); Xiang \& Turner (1995). To characterize the frequency and timescales for maser emission in these YSOs requires multi-epoch observations.

The organization of this contribution will be as follows: first, what can we learn about the stellar or outflow sources by studying water masers ? In particular, what can water masers arond low-luminosity sources reveal about the outflow or the central star ? Second, a review of a program of a monthly monitoring survey of water masers toward low-luminosity YSOs and the results of that survey will be presented. This is important because it characterizes the time scale of water maser activity in the embedded sources. Third, some assorted VLA observations of water masers around these objects will be presented, with a discussion of the position of the water masers with respect to the central energy sources. Finally, a preliminary presentation of VLBA data of the water masers around the YSO IRAS05413-0104, which is the driving source of the molecular hydrogen jet and $\mathrm{H}-\mathrm{H}$ object $\mathrm{HH} 212$ will be made. We will use data from IRAS054130104 for examples of all the water maser observations reported here. Multiple epoch high-resolution observations of these water masers can tell us about the motions of the gas near these young stellar objects.

\section{Why Study Water Masers toward YSOs ?}

Generally speaking, the radiative transfer problem for masers is difficult enough so that one cannot expect to use the observations to determine densities or temperatures of the masing gas, except within some broad bounds. Even if these parameters could be determined, the masers are likely to be found in clumps of gas so that those parameters couldn't be used to make statements about the general physical parameters of the environment 
of the embedded object (at least for the densities). On the other hand, we know that for water masers at $22 \mathrm{GHz}$ to even exist there must be some lower limit to the temperature and densities for the energy levels to be significantly populated. The necessary temperature is $200-300 \mathrm{~K}$, and the $\mathrm{H}_{2}$ densities in the clumps must be in the range $10^{8}-10^{9} \mathrm{~cm}^{-3}$.

But water masers at $22 \mathrm{GHz}$ do give us the ability to do at least three things:

1) Observe the kinematics of the gas where the masers reside, with high velocity resolution $\left(\leq 0.1 \mathrm{~km} \mathrm{~s}^{-1}\right)$ via radio spectroscopy.

2) Observe the kinematics of the gas with high angular resolution using radio interferometry (scales of 1 arcsecond down to 1 mas).

Masers can be used to do these two things for the same reason that we have difficulties in interpreting their physical properties. They are nonlinear processes, so they are very bright and small.

3) Masers also tell us that there are places where water molecules reside at fairly high densities and warm temperatures. These are necessary but not sufficient conditions for water masers, that is, there may be warm, dense clumps of water molecules where masers are not found.

So if we would like to use water masers to study the kinematics of the environment of YSOs at high angular and spectral resolution, we must characterize the emission from a number of objects which we intend to study.

Since their discovery it has been well known that water masers in the interstellar medium are highly variable - why they are is another question which is tied in with the way they are pumped, the radiative transfer, and beaming effects - but they are, and we need to characterize the variability if we are to study kinematics using them.

A number of groups have used single antennas over the years to conduct surveys of star-forming regions in order to characterize the frequency and luminosity with which water masers occur in these regions. Some of these are: Reinhard Genzel and Dennis Downes in the late 1970s generally looked at high-mass star formation regions; Ed Churchwell and his collaborators toward compact and ultra-compact HII regions; and most recently, Marcello Felli and his group have observed low- to mid-luminosity sources. Only in the case of Felli's group has there been a concerted effort to characterize the variability of sources by observing a sample more than once.

\section{Single Antenna Water Maser Monitoring Survey}

After the discovery of water masers toward low-luminosity objects in the $\rho$ Ophiuchus cloud in 1987, and follow-up observations at the VLA by Wilking \& Claussen (1987) and by Terebey, Vogel, \& Myers (1989), we 
realized by the difference in spectra, that to better understand the water masers around low-luminosity objects, we would have to monitor them on monthly time scales over many months to characterize their variability. So we embarked on what we called a monitoring survey; to observe as many sources as possible once per month for as long as we could get time. We have reported on this survey in three recent papers: Wilking et al. (1994a), Wilking et al. (1994b), and Claussen et al. (1996). Here we will summarize and show some data from this survey.

The sample of sources was selected from the IRAS PSC which were known to be associated with molecular outflows and/or dense molecular cores. We also required sources to have rising $\nu \mathrm{S}_{\nu}$ throughout the IRAS bands, to lie in nearby clouds, and to have IRAS in-band luminosities $\leq$ $120 \mathrm{~L}_{\odot}$. Other embedded sources were included such as those revealed by IRAS Pointed Observations, the CPC, and some sources that were confused in the IRAS PSC. We were clearly selecting toward Class I and Class 0 objects.

A list of 45 sources were thus compiled for observations. From these, 27 were monitored on a regular basis which we defined as a minimum of seven observations with no gaps larger than three months. Observations were made using the $37-\mathrm{m}$ radio telescope at Haystack Observatory and the NRAO 43-m radio telescope at Green Bank. Figure 1 shows the spectra of water masers toward IRAS05413-0104 taken over the 13-month period.

The results of the monitoring survey are 1) nine new water maser sources were found, resulting in 24 YSOs with $\mathrm{L} \leq 120 \mathrm{~L}_{\odot}$ known to display water maser activity; 2) the maser phase in low-mass embedded sources is estimated to occupy about one-third the duration of the embedded phase of evolution; 3) some water maser features appear and disappear on timescales less than about 2 months - this characteristic timescale is consistent with shock crossing times for reasonable values of the shock velocity and size; and 4) the isotropic luminosity of the water masers in any given source can vary by at least 2 orders of magnitude over the course of a year - this implies that a single-epoch measurement of the luminosity of the masers may provide a misleading result.

\section{Assorted VLA Observations}

We have used the VLA irregularly, in different configurations making use of bits of telescope time available at the end of software time, etc. in order to refine the positions of the water masers and relate them to other known objects in the field of view such as mm-wave continuum sources, $\mathrm{cm}$-wave continuum sources, IRAS positions, CO sources, etc. Details of these observations and more specific results from them have been and will be reported 


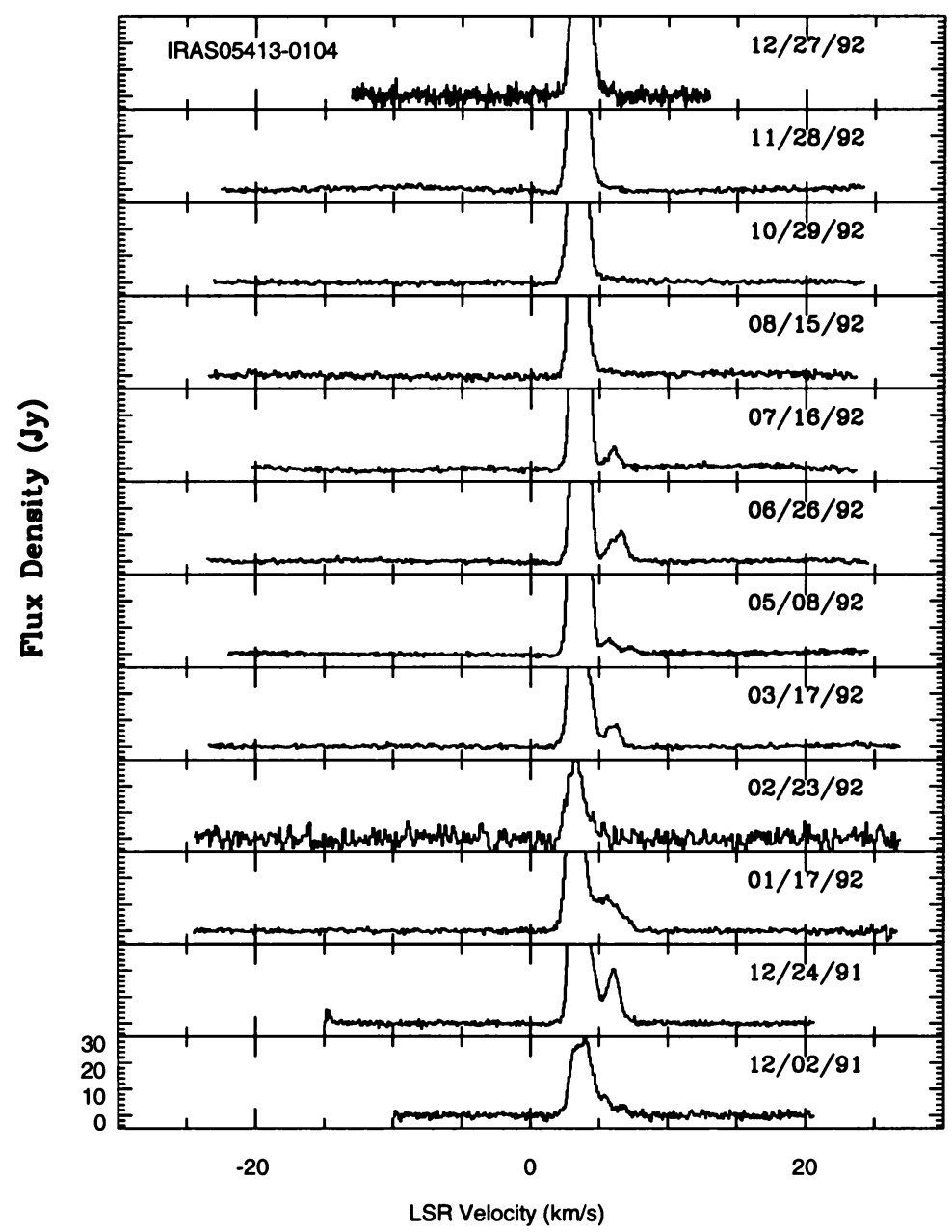

Figure 1. Multi-Epoch Spectra of Water Masers in IRAS05413-0104. The peak flux density for the bright feature is upwards of $300 \mathrm{Jy}$.

elsewhere (Rogers \& Gottschalk 1993; Wootten et al. 1997).

As an example of our VLA data, we show in Figure 2 a picture of IRAS05413-0104, showing the positions of the water masers, the IRAS source, the $3 \mathrm{~mm}$ continuum source, and the centroid of the molecular hydrogen jet. Figure 2 shows that the water masers are clearly associated with the centroid of the molecular hydrogen jet and the $2.7 \mathrm{~mm}$ OVRO continuum source. The water masers, of course give the best positional accuracy of any of these.

The results of these VLA observations point up the fact that, in most 


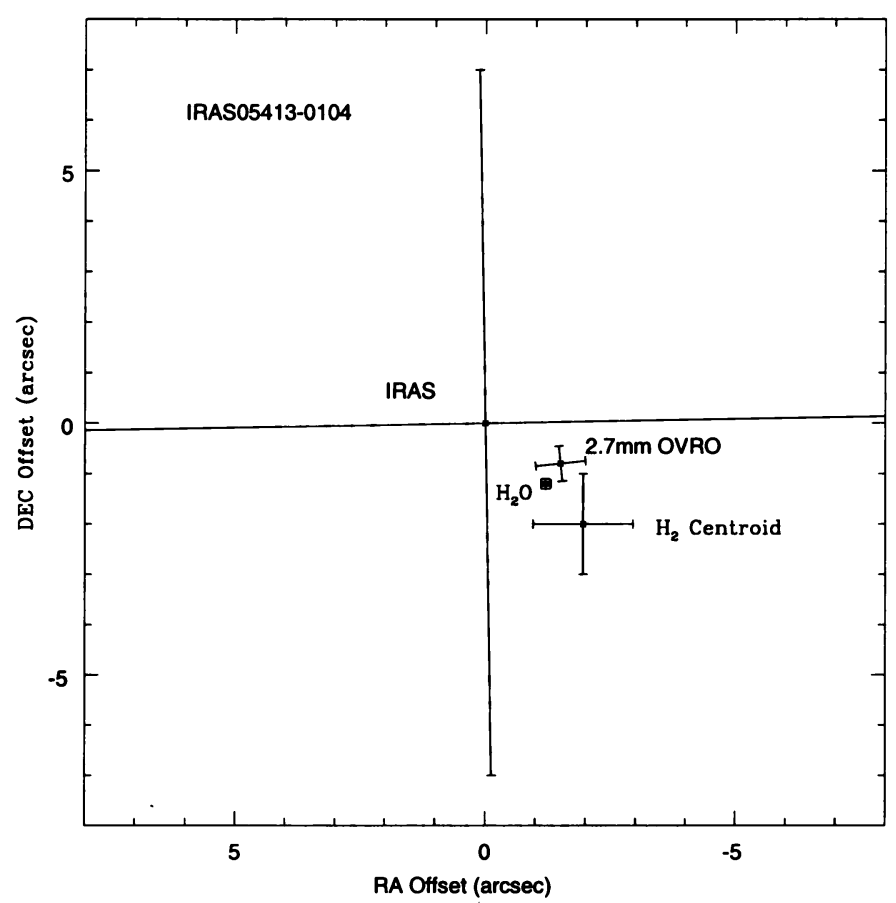

Figure 2. Objects Near the Embedded Source IRAS 05413-0104

sources, the masers all lie within a few hundred A.U. of the stellar object. This suggests that the masers lie in high density gas near the driving source of the outflow, and likely produced in shocks near the star.

\section{VLBA Observations of Water Masers in IRAS05413-0104}

The water masers toward IRAS05413-0104 are one of three maser sources that we detected all the time in our monitoring survey. The masers were usually fairly strong, but IRAS05413-0104 is not one of the most luminous sources in the survey. At $15 \mathrm{~L}_{\odot}$, the bolometric luminosity of the embedded source is more nearly the median in the survey. IRAS05413-0104 is located at the center of a beautiful $\mathrm{H}_{2}$ jet (Zinnecker, McCaughrean, \& Rayner, 1997). There is a $3 \mathrm{~mm}$ radio source at the center as well, and the water masers coincide with the $3 \mathrm{~mm}$ source, midway between the two central $\mathrm{H}_{2}$ knots, which are separated by about 8 arcseconds.

Our VLBA data was taken at four epochs: July 4, July 26, August 16, and September 15, 1996. The entire 10-station array was used, as well as one antenna from the VLA. At $22 \mathrm{GHz}$ the beam size is about 1 mas, 


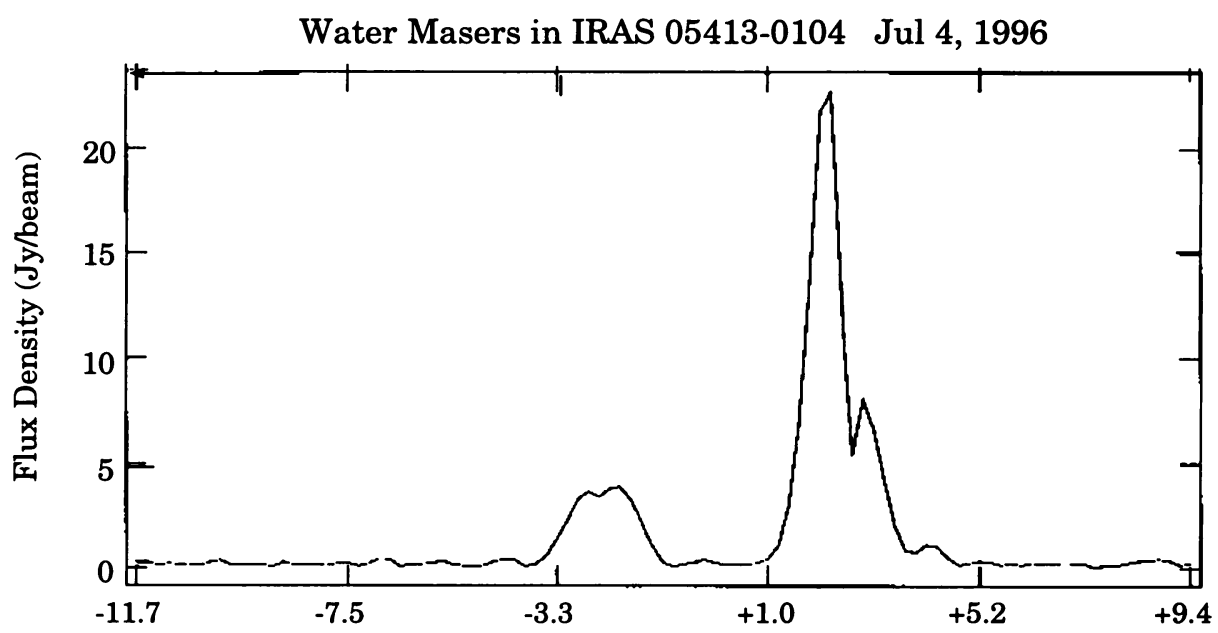

LSR Velocity $(\mathrm{km} / \mathrm{s})$

Figure 3. The Water Maser Spectrum in IRAS 05413-0104 on July 4, 1996

which at the distance of IRAS05413-0104 is 0.5 A.U. At the initial epoch, the spectrum of the water masers looked like Figure 3. Note that the feature that is most blue-shifted is new since 1992, but since we don't have monitoring data since then, we don't know when it appeared or how long it has been around.

When we made the observations, we expected to see several maser "spots", or features. What we saw is shown in Figure 4 - there are more than 30 distinct features here. The first thing to notice from Figure 4 is that the masers lie mostly along a line that has a position angle $(\mathrm{N}$ through E) almost exactly the same as the position angle of the jet (Zinnecker, McCaughrean, \& Rayner, 1997). This suggests that the masers are tracing the outflow very near to the star, rather than lying in a disk around the star. The blue-shifted features (at $\sim-3 \mathrm{~km} \mathrm{~s}^{-1}$; see Figure 3 ) are all found in the group of masers in the northeast of the map of Figure 4. The most extreme red-shifted emission (at $\sim 5 \mathrm{~km} \mathrm{~s}^{-1}$ ) are found as the compact clump of features just south and east of the $(0,0)$ position. The strong, intermediate velocity feature relates to the linear string of masers at the $(0,0)$ position in Figure 4.

In Figure 5, maps of the blue-shifted masers are shown for all four epochs. The very high resolution available by observing water masers with the VLBA is clear from this figure, as well as the definite changes in the details of the structure, although the general structure over 130 A.U. is 


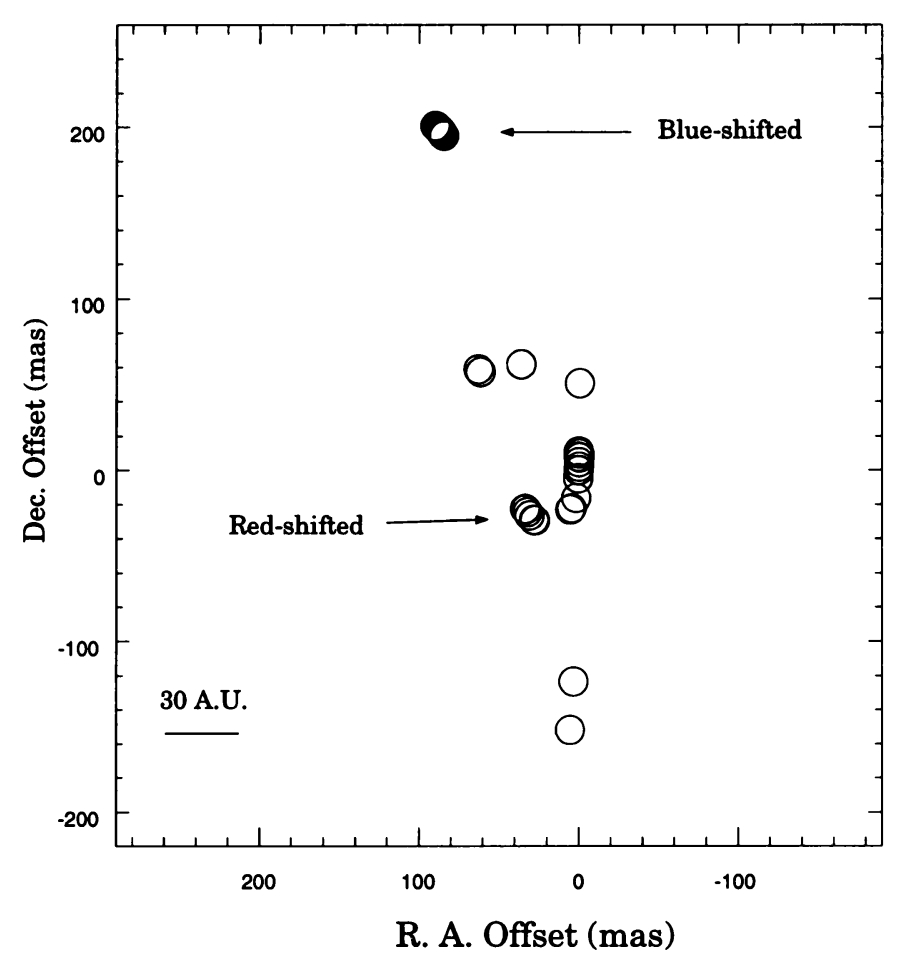

Figure 4. Water Maser Positions near IRAS 05413-0104 on July 4, 1996.

very similar over all four epochs. The proper motion detectable by relative measurements over one month interval is $25 \mathrm{~km} \mathrm{~s}^{-1}$ for a distance of $500 \mathrm{pc}$. We are currently analysing the details of the proper motions of the maser spots and the results for IRAS05413-0104 will be published soon (Claussen et al., 1997).

\section{Summary}

We have discussed the importance of maser observations, both as single antenna monitoring observations, and high resolution $\sim 1$ mas monitoring observations, to understand the structure of the gas within a few A.U. of the embedded sources. Water masers are a very valuable probe of the circumstellar or near stellar environment. Further studies will allow us to place the masers in a circumstellar disk or at the very base of the outflow.

Acknowledgements: The VLA and the VLBA are instruments operated by the National Radio Astronomy Observatory, which is a facility of the National Science Foundation under cooperative agreement by As- 

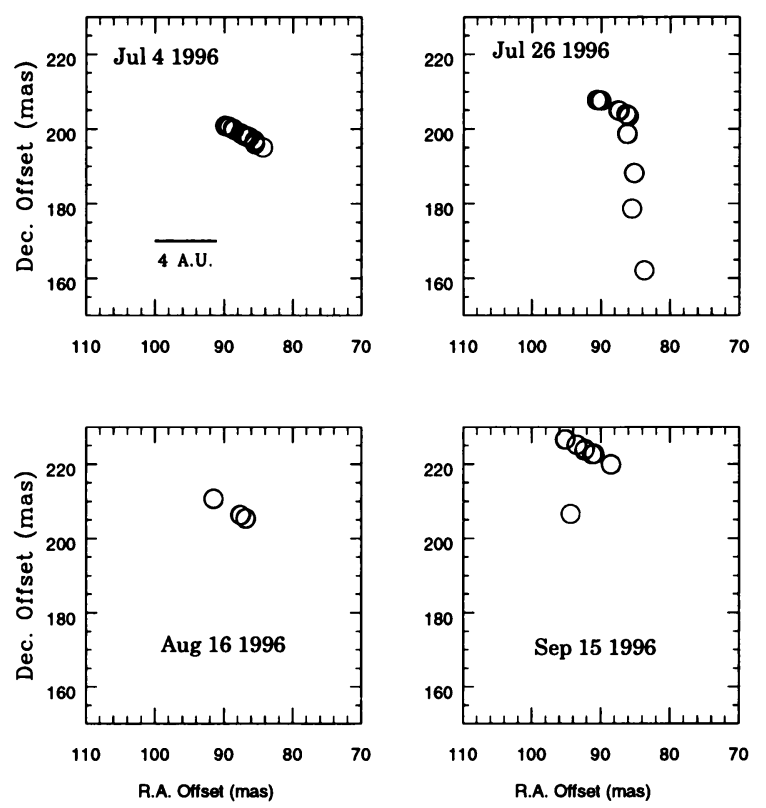

Figure 5. Blueshifted Water Maser Positions near IRAS 05413-0104 four Four Epochs

sociated Universities, Inc. The authors would like to thank the Scientific Organizing Committee for providing the opportunity to present these results at the conference.

\section{References}

Claussen, M. J, Wilking, B. A., Benson, P. J., Wootten, H. A., Myers, P. C., \& Terebey, S. 1996, ApJ Supp. Series, 106, 111

Claussen, M. J, Marvel, K. B., Wootten, H. A., \& Wilking, B. A. 1997, in preparation

Comoretto, G., it et al. 1990, A\&A Supp. Series, 84, 179

Genzel, R., \& Downes, D. 1977, A\&A Supp. Series, 30, 145

Felli, M., Palagi, F., \& Tofani, G. 1992, A\&A, 255, 293

Rodriguez, L. F., Moran, J. M., Ho, P. T. P., \& Gottlieb, E. W., 1980, ApJ 235, 845

Rogers, C. B. \& Gottschalk, J. 1993, B.A.A.S., 25, 1367

Terebey, S., Vogel, S. N., \& Myers, P. C. 1989, ApJ, 340, 472

Wilking, B. A. \& Claussen, M. J. 1987, ApJL, 320, L133

Wilking, B. A., Claussen, M. J., Benson, P. J., Myers, P. C., Terebey, S., \& Wootten, H. A. 1994, ApJ, 431, L119

Wilking, B. A. \& Claussen, M. J., Benson, P. J., Myers, P. C., Terebey, S., \& Wootten, H. A. 1994b, in Clouds, Cores, and Low-Mass Stars, eds. D. P. Clemens \& R. Barvainis (ASP: San Francisco), p. 299 
Wootten, H. A., Wilking, B. A., Meehan, L. \& Claussen, M. J 1997, in preparation

Xiang, D. \& Turner, B. E. 1992, Acta Astronomica, 33, 87

Xiang, D. \& Turner, B. E. 1995, ApJ Supp. Series, 99, 121

Zinnecker, H., McCaughrean, M., \& Rayner, J., in Low Mass Star Formation from Infall to Outflow, ed. F. Malbet \& A. Castets 1997, IAU Symposium No. 182. 